\title{
A Product of Theta-Functions Analogous to Ramanujan's Remarkable Product of Theta-Functions and Applications
}

\author{
Nipen Saikia \\ Department of Mathematics, Rajiv Gandhi University, Rono Hills, Doimukh 791112, Arunachal Pradesh, India \\ Correspondence should be addressed to Nipen Saikia; nipennak@yahoo.com
}

Received 24 September 2012; Accepted 12 November 2012

Academic Editor: Andrea Pascucci

Copyright (C) 2013 Nipen Saikia. This is an open access article distributed under the Creative Commons Attribution License, which permits unrestricted use, distribution, and reproduction in any medium, provided the original work is properly cited.

We define a product $l_{k, n}$ for any positive real numbers $k$ and $n$ involving Ramanujan's theta-functions $\phi(q)$ and $\psi(q)$ which is analogous to Ramanujan's remarkable product of theta-functions recorded by Ramanujan (1957) and study its several properties. We prove general theorems for the explicit evaluations of $l_{k, n}$ and find some explicit values. As application of the product $l_{k, n}$, we also offer explicit formulas for explicit values of Ramanujan's continued fraction $V(q)$ in terms of $l_{k, n}$ and give examples.

\section{Introduction}

Ramanujan's theta-functions $\phi(q), \psi(q)$, and $f(-q)$ are defined as

$$
\begin{gathered}
\phi(q):=\sum_{n=-\infty}^{\infty} q^{n^{2}}=\frac{(-q ;-q)_{\infty}}{(q ;-q)_{\infty}}, \\
\psi(q):=\sum_{n=0}^{\infty} q^{n(n+1) / 2}=\frac{\left(q^{2} ; q^{2}\right)_{\infty}}{\left(q ; q^{2}\right)_{\infty}}, \\
f(-q):=f\left(-q,-q^{2}\right)=\sum_{n=-\infty}^{\infty}(-1)^{n} q^{n(3 n-1) / 2}=(q ; q)_{\infty},
\end{gathered}
$$

where $(a ; q)_{\infty}:=\prod_{k=0}^{\infty}\left(1-a q^{k}\right)$.

On page 338 of his first notebook, Ramanujan [1] defined the remarkable product of theta-functions as

$$
a_{m, n}=n e^{-(\pi / 4)(n-1) \sqrt{m / n}} \frac{\psi^{2}\left(e^{-\pi \sqrt{m n}}\right) \phi^{2}\left(-e^{-2 \pi \sqrt{m n}}\right)}{\psi^{2}\left(e^{-\pi \sqrt{m / n}}\right) \phi^{2}\left(-e^{-2 \pi \sqrt{m / n}}\right)},
$$

where $m$ and $n$ are positive real numbers. He then, on pages 338 and 339, offered a list of eighteen particular values of the product $a_{m, n}$. All these eighteen values are proved by Berndt et al. [2]. An account of these can be found in Berndt's book [3]. Naika and Dharmendra [4] also established some general theorems for explicit evaluations of the product $a_{m, n}$ and found some new explicit values therefrom. Further results on $a_{m, n}$ can be found in $[5,6]$.

In [7], Mahadeva Naika et al. defined the product

$$
b_{m, n}=n e^{-(\pi / 4)(n-1) \sqrt{m / n}} \frac{\psi^{2}\left(-e^{-\pi \sqrt{m n}}\right) \phi^{2}\left(-e^{-2 \pi \sqrt{m n}}\right)}{\psi^{2}\left(-e^{-\pi \sqrt{m / n}}\right) \phi^{2}\left(-e^{-2 \pi \sqrt{m / n}}\right)} .
$$

They established general theorems for explicit evaluations of $b_{m, n}$ and obtained some particular values. Mahadeva Naika et al. [8] established general formulas for explicit values of Ramanujan's cubic continued fraction $V(q)$ in terms of the products $a_{m, n}$ and $b_{m, n}$ defined above, where

$$
V(q):=\frac{q^{1 / 3}}{1}+\frac{q+q^{2}}{1}+\frac{q^{2}+q^{4}}{1}+\frac{q^{3}+q^{6}}{1}+\cdots, \quad|q|<1 .
$$

and found some particular values of $V(q)$.

Motivated by the above work, in this paper, we define the product of theta-functions $l_{k, n}$ as

$$
l_{k, n}=\frac{\psi(-q) \phi\left(q^{k}\right)}{q^{(k-1) / 8} \psi\left(-q^{k}\right) \phi(q)} ; \quad q:=e^{-\pi \sqrt{n / k}},
$$

where $n$ and $k$ are positive real numbers. We establish several properties of the product $l_{k, n}$. We prove general formulas for explicit evaluations of $l_{k, n}$ and find its explicit values. As 
application of the product $l_{k, n}$, we also offer explicit formulas for explicit values of Ramanujan's cubic continued fraction $V(q)$ in terms of $l_{k, n}$ and give examples.

In Section 2, we collect some preliminary results. In Section 3, we prove several properties of the product $l_{k, n}$. Section 4 , is devoted to find explicit values of $l_{k, n}$. Finally in Section 5, we offer explicit formulas for explicit evaluations of continued fraction $V(q)$ in terms of $l_{k, n}$ with examples.

To end this introduction, we define Ramanujan's modular equation. Let $K, K^{\prime}, L$, and $L^{\prime}$ denote the complete elliptic integrals of the first kind associated with the moduli $k, k^{\prime}, l$, and $l^{\prime}$, respectively. Suppose that the equality

$$
n \frac{K^{\prime}}{K}=\frac{L^{\prime}}{L}
$$

holds for some positive integer $n$. Then a modular equation of degree $n$ is a relation between the moduli $k$ and $l$ which is implied by (7). Ramanujan recorded his modular equations in terms of $\alpha$ and $\beta$, where $\alpha=k^{2}$ and $\beta=l^{2}$. We say that $\beta$ has degree $n$ over $\alpha$. By denoting $z_{r}=\phi^{2}\left(q^{r}\right)$, where $q=$ $\exp \left(-\pi K^{\prime} \mid K\right),|q|<1$, the multiplier $m$ connecting $\alpha$ and $\beta$ is defined by $m=z_{1} / z_{n}$.

\section{Preliminary Results}

Lemma 1 (see [9, p. 43, Entry 27(i)]). If $\alpha \beta=\pi$ then

$$
\sqrt{\alpha} \phi\left(e^{-\alpha^{2}}\right)=\sqrt{\beta} \phi\left(e^{-\beta^{2}}\right)
$$

Lemma 2 (see $[10$, p. $1049,(1.13)])$. If $\alpha \beta=\pi^{2}$ then

$$
e^{-\alpha / 8} \sqrt[4]{\alpha} \psi\left(-e^{-\alpha}\right)=e^{-\beta / 8} \sqrt[4]{\beta} \psi\left(-e^{-\beta}\right)
$$

Lemma 3 (see [9, p. 122, Entry 10(i)]). One has

$$
\phi(q)=\sqrt{z_{1}} .
$$

Lemma 4 (see [9, p. 123, Entry 11(ii)]). One has

$$
\psi(-q)=\sqrt{\frac{z_{1}}{2}}\{\alpha(1-\alpha)\}^{1 / 8} q^{-1 / 8}
$$

Lemma 5 (see [9, p. 124, Entry 12(v), (vi)]). One has

$$
\begin{aligned}
& \chi(q)=2^{1 / 6}\left\{\frac{\alpha(1-\alpha)}{q}\right\}^{-1 / 24}, \\
& \chi(-q)=2^{1 / 6}(1-\alpha)^{1 / 12}\left(\frac{\alpha}{q}\right)^{-1 / 24}
\end{aligned}
$$

One also notes that if we replace $q$ by $q^{n}$ in the Lemmas 3 and 4 , then $z_{1}$ and $\alpha$ will be replaced by $z_{n}$ and $\beta$, respectively, where $\beta$ has degree $n$ over $\alpha$.
Lemma 6 (see $[9$, p. 345, Entry 1(i), (ii)]). If $V(q)$ is as defined in (5), then

$$
\begin{aligned}
& 1+\frac{1}{V(q)}=\frac{\psi\left(q^{1 / 3}\right)}{q^{1 / 3} \psi\left(q^{3}\right)}, \\
& 1+\frac{1}{V^{3}(q)}=\frac{\psi^{4}(q)}{q \psi^{4}\left(q^{3}\right)}, \\
& 2 V(q)=1-\frac{\phi\left(-q^{1 / 3}\right)}{\phi\left(-q^{3}\right)} .
\end{aligned}
$$

Lemma 7 (see [9, p. 347]). If $V(q)$ is as defined in (5), then

$$
1-8 V^{3}(q)=\frac{\phi^{4}(-q)}{\phi^{4}\left(-q^{3}\right)} .
$$

Lemma 8 (see $[9$, p. 231, Entry 5(xii) $]$ ). Let $P=$ $\{16 \alpha \beta(1-\alpha)(1-\beta)\}^{1 / 8}$ and $Q=(\beta(1-\beta) / \alpha(1-\alpha))^{1 / 4}$, then

$$
Q+Q^{-1}+2 \sqrt{2}\left(P-P^{-1}\right)=0
$$

where $\beta$ has degree 3 over $\alpha$.

Lemma 9 (see $[9$, p. 282, Entry 13(xiv)]). Let $P=$ $\{16 \alpha \beta(1-\alpha)(1-\beta)\}^{1 / 12}$ and $Q=(\beta(1-\beta) / \alpha(1-\alpha))^{1 / 8}$, then

$$
Q+Q^{-1}+2\left(P-P^{-1}\right)=0
$$

where $\beta$ has degree 5 over $\alpha$.

\section{Some Properties of $l_{k, n}$}

In this section, we study some properties of the product $l_{k, n}$.

Theorem 10. For all positive real numbers $k$ and $n$, one has
(i) $l_{k, 1}=1$,
(ii) $l_{k, 1 / n}=1 / l_{k, n}$,
(iii) $l_{k, n}=l_{n, k}$.

Proof. Using the definition of $l_{k, n}$ and Lemmas 1 and 2, we easily arrive at (i). Replacing $n$ by $1 / n$ in $l_{k, n}$ and using Lemmas 1 and 2, we find that $l_{k, n} l_{k, 1 / n}=1$ which completes the proof of (ii). To prove (iii), we interchange $n$ and $k$ in $l_{k, n}$.

Remark 11. By using the definitions of $\phi(q), \psi(q)$, and $l_{k, n}$, it can be seen that $l_{k, n}$ has positive real value and that the values of $l_{k, n}$ increases as $n$ increases when $k>1$. Thus, by Theorem 10(i), $l_{k, n}>1$ for all $n>1$ if $k>1$.

Theorem 12. For all positive real numbers $k, m$, and $n$, one has

$$
l_{k, n / m}=\frac{l_{m k, n}}{l_{n k, m}} .
$$


Proof. Using the definition of $l_{k, n}$, we find that

$$
\frac{l_{m k, n}}{l_{n k, m}}=e^{-\pi(\sqrt{n / m k}-\sqrt{m / n k}) / 8} \frac{\psi\left(-e^{-\pi \sqrt{n / m k}}\right) \phi\left(e^{-\pi \sqrt{m / n k}}\right)}{\psi\left(-e^{-\pi \sqrt{m / n k}}\right) \phi\left(e^{-\pi \sqrt{n / m k}}\right)} .
$$

Applying Lemmas 1 and 2 in the denominator of right hand side of (20) and simplifying using Theorem 10(ii) and (iii), we complete the proof.

Corollary 13. For all positive real numbers $k$ and $n$, one has

$$
l_{k^{2}, n}=l_{n k, n} l_{k, n / k}
$$

Proof. Setting $k=n$ in Theorem 12 and simplifying using Theorem 10(ii), we obtain

$$
l_{k^{2}, m}=l_{m k, k} l_{k, m / k}
$$

Replacing $m$ by $n$, we complete the proof.

Theorem 14. Let $k, a, b, c$, and $d$ be positive real numbers such that $a b=c d$. Then

$$
l_{a, b} l_{k c, k d}=l_{k a, k b} l_{c, d} .
$$

Proof. From the definition of $l_{k, n}$ and using $a b=c d$ for positive real numbers $k, a, b, c$, and $d$, we deduce that

$$
l_{k a, k b} l_{a, b}^{-1}=l_{k c, k d} l_{c, d}^{-1} \text {. }
$$

Rearranging the terms in (24) we arrive at the desired result.

Corollary 15. For any positive real numbers $n$ and $p$, one has

$$
l_{n p, n p}=l_{n p^{2}, n} l_{p, p} .
$$

Proof. The result follows immediately from Theorem 14 with $a=p^{2}, b=1, c=d=p$ and $k=n$.

Theorem 16. For all positive real numbers $a, b, c$, and $d$, one has

$$
l_{a / b, c / d}=\frac{l_{a d, b c}}{l_{a c, b d}}
$$

Proof. Applying Theorem 10(iii) in Theorem 12, we deduce that, for all positive real numbers $a, b$, and $n$

$$
l_{a / b, n}=l_{a, b n} l_{b, a n}^{-1} .
$$

Now setting $n=c / d$ and again employing Theorems 12 and 10 (iii) in (27), we complete the proof.

Theorem 17. For all positive real numbers $a$ and $b$, one has

(i) $l_{a / b, a / b}=l_{b, b} l_{a, a / b^{2}}$,

(ii) $l_{a, a} l_{a, b^{2} / a}=l_{b, b} l_{b, a^{2} / b}$,

(iii) $l_{a, a} l_{b, a^{2} b}=l_{b, b a, a b^{2}}$.
Proof. By using Theorems 10(ii) and 16, we find that

$$
l_{a / b, a / b}=l_{b, b} l_{a, a / b^{2}}
$$

So we complete the proof of (i). Setting $n=a / b$ in (27), we find that

$$
l_{a / b, a / b}=l_{a, a} l_{b, a^{2} / b}^{-1} .
$$

Now (ii) follows from (28) and (29) and Theorem 10(ii).

By using Theorems 10(ii) and 12, we find that

$$
l_{a / b, a / b}=l_{b, b} l_{a b^{2}, a} l_{a^{2}, b^{2}}^{-1} .
$$

Similarly, we find that

$$
l_{b / a, b / a}=l_{a, a} l_{a^{2} b, b} l_{b^{2}, a^{2}}^{-1} .
$$

From (30), (31), and Theorem 10(ii) and (iii), we complete the proof of (iii).

\section{Explicit Values of $l_{k, n}$}

In this section, we prove general theorems for explicit evaluations of $l_{k, n}$ and find some explicit values. First we define Ramanujan's class invariants. Ramanujan's two class invariants $G_{n}$ and $g_{n}$ which are defined by

$$
\begin{gathered}
G_{n}=2^{-1 / 4} q^{-1 / 24} \chi(q), \\
g_{n}=2^{-1 / 4} q^{-1 / 24} \chi(-q), \quad q:=e^{-\pi \sqrt{n}},
\end{gathered}
$$

where $\chi(q)=\left(-q ; q^{2}\right)_{\infty}$ and $n$ is a positive rational number. Employing Lemma 5 in (32) it follows that

$$
\begin{gathered}
G_{n}=\{4 \alpha(1-\alpha)\}^{-1 / 24}, \\
g_{n}=2^{-1 / 12}(1-\alpha)^{1 / 12} \alpha^{-1 / 24} .
\end{gathered}
$$

Also, if $\beta$ has degree $r$ over $\alpha$, then

$$
\begin{gathered}
G_{r^{2} n}=\{4 \beta(1-\beta)\}^{-1 / 24}, \\
g_{r^{2} n}=2^{-1 / 12}(1-\beta)^{1 / 12} \beta^{-1 / 24} .
\end{gathered}
$$

In his notebooks [1] and paper [11], Ramanujan recorded a total of 116 class invariants or monic polynomials satisfied by them. An account of these can also be found in Berndt's book [3]. For further references, see [2, 12-17].

Theorem 18. One has

$$
l_{k, n}=\left(\frac{G_{n k}}{G_{n / k}}\right)^{3} .
$$

Proof. Employing Lemmas 3 and 4 in the definition of $l_{k, n}$, we find that

$$
l_{k, n}=\left(\frac{\alpha(1-\alpha)}{\beta(1-\beta)}\right)^{1 / 8}
$$


Again from (32) and (33) with $q:=e^{-\pi \sqrt{n / k}}$, we find that

$$
\frac{G_{n k}}{G_{n / k}}=\left(\frac{\alpha(1-\alpha)}{\beta(1-\beta)}\right)^{1 / 24}
$$

Combining (36) and (37), we complete the proof.

From Theorem 18, it is obvious that if we know $\left(G_{n k} / G_{n / k}\right)$ or $\left(G_{n k}\right.$ and $\left.G_{n / k}\right)$ then explicit values of $l_{k, n}$ can easily be determined. For example, we find some values of $l_{k, n}$ in next theorem by employing the values of $\left(G_{n k} / G_{n / k}\right)$ or $\left(G_{n k}\right.$ and $\left.G_{n / k}\right)$ from literature.

Theorem 19. One has

(i) $l_{3,5}=\left(G_{15} G_{5 / 3}\right)^{3}=\left(\frac{3+\sqrt{5}}{2}\right)$,

(ii) $l_{3,7}=\left(G_{21} / G_{7 / 3}\right)^{3}=(3 \sqrt{3}+2 \sqrt{7})^{1 / 2}$,

(iii) $l_{3,11}=\left(G_{33} / G_{11 / 3}\right)^{3}=(3+\sqrt{11} / \sqrt{2})$,

(iv) $l_{3,13}=\left(G_{39} / G_{13 / 3}\right)^{3}=1 / 2^{3 / 2}((3+\sqrt{13}) / 2+$ $\left.\sqrt{(3+3 \sqrt{13}) / 2}^{3 / 2}\right)$,

(v) $l_{5,11}=\left(G_{55} / G_{11 / 5}\right)^{3}=1 / 2((9+5 \sqrt{5}) / 2+$ $\sqrt{(95+45 \sqrt{5}) / 2)}$,

(vi) $l_{5,13}=\left(G_{65} / G_{13 / 5}\right)^{3}=(\sqrt{(7+\sqrt{65}) / 8}+$ $\sqrt{(\sqrt{65}-1) / 8)^{3}}$

(vii) $l_{3,23}=\left(G_{69} / G_{23 / 3}\right)^{3}=(24+5 \sqrt{23})^{1 / 4}(25+$ $3 \sqrt{69} / 2)^{3 / 8}$

(viii) $l_{3,47}=\left(G_{141} / G_{47 / 3}\right)^{3}=(48+7 \sqrt{47})^{3 / 12}(95+$ $8 \sqrt{141})^{3 / 8}$,

(ix) $l_{3,71}=\left(G_{213} / G_{71 / 3}\right)^{3}=\left(3480+413 \sqrt{71}^{1 / 4}((73+\right.$ $5 \sqrt{213}) / 2)^{3 / 8}$.

Proof. For (i)-(vi), the corresponding values of $\left(G_{n k} / G_{n / k}\right)$ can be found in equations (4.10), (4.20), (4.29), (4.37), (4.51), and (4.60), respectively of [12]. For (vii) to (ix), the corresponding values of can be found in equations [p. 227, (5.8)], [p. 229, (5.15)], and [p. 233, (5.25)], respectively of [3].

Corollary 20. One has

$$
l_{n, n}=\left(G_{n^{2}}\right)^{3} .
$$

Proof. We set $k=n$ in Theorem 18 and use the result $G_{1}=1$, to complete the proof.

From Corollary 20, it is obvious explicit values of $l_{n, n}$ can easily be determined if the corresponding values of the class invariants $G_{n^{2}}$ are known. We give some examples in the next theorem.
Theorem 21. One has

(i) $l_{2,2}=G_{4}^{3}=(1+\sqrt{2})^{3 / 4} / 2^{9 / 16}$,

(ii) $l_{4,4}=G_{16}^{3}=2^{-3 / 4}\left(16+152^{1 / 4}+12 \sqrt{2}+92^{3 / 4}\right)^{3}$,

(iii) $l_{3,3}=G_{9}^{3}=(1+\sqrt{3}) / \sqrt{2}$,

(iv) $l_{5,5}=G_{25}^{3}=\left(\left(7^{1 / 4}+\sqrt{4+\sqrt{7}}\right) /(2)\right)^{3}$,

(v) $l_{7,7}=G_{49}^{3}=((1+\sqrt{5}) /(2))^{3}$,

(vi) $l_{9,9}=G_{81}^{3}=(\sqrt[3]{2(\sqrt{3}+1)}+1) /(\sqrt[3]{2(\sqrt{3}-1)}+1)$.

Proof. For (i) and (ii), we use the values of $G_{4}$ and $G_{16}$ from [18, p. 114-115, Theorem 6.2.2(ii) (vi)]. For (iii)-(vi), we use the corresponding values of $G_{n}$ from [3, p. 189-193].

Ramanujan's Schalfli type modular equations of prime degree can also be used to explicit values of the product $l_{k, n}$. We offer two theorems as examples.

Theorem 22. One has

$$
l_{3, n}^{-2}+l_{3, n}^{2}+2 \sqrt{2}\left\{\left(G_{3 n} G_{n / 3}\right)^{-3}-\left(G_{3 n} G_{n / 3}\right)^{3}\right\}=0 .
$$

Proof. We use the definitions of class invariants $l_{k, n}$ and $G_{n}$ in Lemma 8 , to complete the proof.

Theorem 23. One has

$$
l_{5, n}+l_{5, n}^{-1}+2\left\{\left(G_{5 n} G_{n / 5}\right)^{-2}-\left(G_{5 n} G_{n / 5}\right)^{2}\right\}=0 .
$$

Proof. We use the definitions of class invariants $l_{k, n}$ and $G_{n}$ in Lemma 9, to complete the proof.

\section{Evaluations of Ramanujan's Cubic Continued Fraction}

In this section, we prove two formulas of explicit evaluations Ramanujan's cubic continued fraction $V(q)$ in terms of the product $l_{k, n}$. We also give examples.

Theorem 24. One has

$$
V^{3}\left(-e^{-\pi \sqrt{n / 3}}\right)=\frac{l_{3, n}^{4}+1-\sqrt{l_{3, n}^{8}+34 l_{3, n}^{4}+1}}{16 l_{3, n}^{4}} .
$$

Proof. Replacing $q$ by $-q$ in (14) and Lemma 7 , we obtain

$$
\begin{gathered}
-\left(1+\frac{1}{V^{3}(q)}\right)=\frac{\psi^{4}(-q)}{q \psi^{4}\left(-q^{3}\right)}, \\
1-8 V^{3}(-q)=\frac{\phi^{4}(q)}{\phi^{4}\left(q^{3}\right)} .
\end{gathered}
$$

Dividing (42) by (43), setting $q:=e^{-\pi \sqrt{n / 3}}$ and simplifying using the definition of $l_{k, n}$, we obtain

$$
8 l_{3,4}^{4} V^{6}\left(-e^{-\pi \sqrt{n / 3}}\right)-\left(l_{3,4}^{4}+1\right) V^{3}\left(-e^{-\pi \sqrt{n / 3}}\right)-1=0 .
$$


Solving (2) for $V^{3}\left(-e^{-\pi \sqrt{n / 3}}\right)$ and noting that $V\left(-e^{-\pi \sqrt{n / 3}}\right)<0$ and the fact in Remark 11, we arrive at the desired result.

From Theorem 24, it is clear that to find explicit values of $V^{3}\left(-e^{-\pi \sqrt{n / 3}}\right)$ it is enough to know the explicit values of $l_{3, n}$. For example, noting $l_{3,1}=1$ from Theorem 10(i) we evaluate

$$
V^{3}\left(-e^{-\pi / \sqrt{3}}\right)=-1 / 4
$$

Theorem 25. One has

$$
V\left(-e^{-\pi \sqrt{n}}\right)=\frac{l_{9, n}+1-\sqrt{l_{9, n}^{2}+10 l_{9, n}+1}}{4 l_{9, n}} .
$$

Proof. Replacing $q$ by $q^{3}$ and then replacing $q$ by $-q$ in (13) and (15), we obtain

$$
\begin{aligned}
-\left(1+\frac{1}{V\left(-q^{3}\right)}\right) & =\frac{\psi(-q)}{q \psi\left(-q^{9}\right)}, \\
1-2 V\left(-q^{3}\right) & =\frac{\phi(q)}{\phi\left(q^{9}\right)} .
\end{aligned}
$$

Dividing (47) by (48), setting $q:=e^{-\pi \sqrt{n / 9}}$ and simplifying by using the definition of $l_{k, n}$, we arrive at

$$
2 l_{9, n} V^{2}\left(-e^{-\pi \sqrt{n}}\right)-\left(l_{9, n}+1\right) V\left(-e^{-\pi \sqrt{n}}\right)-1=0 .
$$

Solving (49) for $V\left(-e^{-\pi \sqrt{n}}\right)$ and noting $V\left(-e^{-\pi \sqrt{n}}\right)<0$ and the fact in Remark 11, we complete the proof.

From Theorem 25, it is obvious that if we know the values of $l_{9, n}$ the corresponding values of $V\left(-e^{-\pi \sqrt{n}}\right)$ can easily be evaluated. For example, by noting $l_{9,1}=1$ from Theorem 10(i) we calculate

$$
V\left(-e^{-\pi}\right)=\frac{1-\sqrt{3}}{2} .
$$

Similarly, one can find explicit values of $V\left(-e^{-3 \pi}\right)$ by using the value of $l_{9,9}$ from Theorem 21 (vi).

\section{Acknowledgments}

The author is thankful to University Grants Commission, New Delhi, India for partially supporting the research work under the grant no. F. No. 41-1394/2012(SR).

\section{References}

[1] S. Ramanujan, Notebooks, vol. 1-2, Tata Institute of Fundamental Research, Bombay, India, 1957.

[2] B. C. Berndt, H. H. Chan, and L.-C. Zhang, "Ramanujan's remarkable product of theta-functions," Proceedings of the Edinburgh Mathematical Society, vol. 40, no. 3, pp. 583-612, 1997.

[3] B. C. Berndt, Ramanujan's Notebooks. Part V, Springer, New York, NY, USA, 1998.
[4] M. S. M. Naika and B. N. Dharmendra, "On some new general theorems for the explicit evaluations of Ramanujans remarkable product of theta-functions," The Ramanujan Journal, vol. 15, no. 3, pp. 349-366, 2008.

[5] M. S. M. Naika, B. N. Dharmendra, and K. Shivashankara, "On some new explicit evaluations of Ramanujans remarkable product of theta-function," The South East Asian Journal of Mathematics and Mathematical Sciences, vol. 5, no. 1, pp. 107-119, 2006.

[6] M. S. M. Naika and M. C. Maheshkumar, "Explicit evaluations of Ramanujans remarkable product of theta-function," Advanced Studies in Contemporary Mathematics, vol. 13, no. 2, pp. 235-254, 2006.

[7] M. S. Mahadeva Naika, M. C. Maheshkumar, and K. S. Bairy, "On some remarkable product of theta-function," The Australian Journal of Mathematical Analysis and Applications, vol. 5, no. 1, pp. 1-15, 2008.

[8] M. S. N. Mahadeva Naika, M. C. S. Maheshkumar, and K. S. Bairy, "General formulas for explicit evaluations of Ramanujan's cubic continued fraction," Kyungpook Mathematical Journal, vol. 49, no. 3, pp. 435-450, 2009.

[9] B. C. Berndt, Ramanujan's Notebooks. Part III, Springer, New York, NY, USA, 1991.

[10] C. Adiga, T. Kim, M. S. Mahadeva Naika, and H. S. Madhusudhan, "On Ramanujan's cubic continued fraction and explicit evaluations of theta-functions," Indian Journal of Pure and Applied Mathematics, vol. 35, no. 9, pp. 1047-1062, 2004.

[11] S. Ramanujan, "Modular equations and approximations to $\pi$," Quarterly Journal of Mathematics, vol. 45, pp. 350-372, 1914.

[12] N. D. Baruah, "On some class invariants of Ramanujan," The Journal of the Indian Mathematical Society, vol. 68, no. 1-4, pp. 113-131, 2001.

[13] B. C. Berndt and H. H. Chan, "Some values for the RogersRamanujan continued fraction," Canadian Journal of Mathematics. (Journal Canadien de Mathématiques), vol. 47, no. 5, pp. 897-914, 1995.

[14] B. C. Berndt, H. H. Chan, and L.-C. Zhang, "Ramanujan's class invariants with applications to the values of $q$-continued fractions and theta functions," in Special Functions, q-Series and Related Topics, M. Ismail, D. Masson, and M. Rahman, Eds., vol. 14, pp. 37-53, American Mathematical Society, Providence, RI, USA, 1997.

[15] B. C. Berndt, H. H. Chan, and L. C. Zhang, "Ramanujan's class invariants and cubic continued fraction," Acta Arithmetica, vol. 73, no. 1, pp. 67-85, 1995.

[16] B. C. Berndt, H. H. Chan, S.-Y. Kang, and L.-C. Zhang, "A certain quotient of eta-functions found in Ramanujan's lost notebook," Pacific Journal of Mathematics, vol. 202, no. 2, pp. 267-304, 2002.

[17] N. Saikia, "Ramanujan's modular equations and WeberRamanujan class invariants $G_{n}$ and $g_{n}$," Bulletin of Mathematical Sciences, vol. 2, no. 1, pp. 205-223, 2012.

[18] J. Yi, Construction and application of modular equations [Ph.D. thesis], University of Illinois at Urbana Champaign, 2004. 


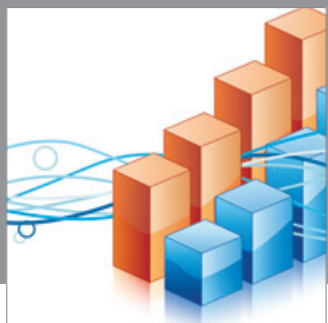

Advances in

Operations Research

mansans

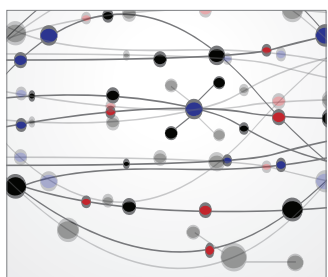

The Scientific World Journal
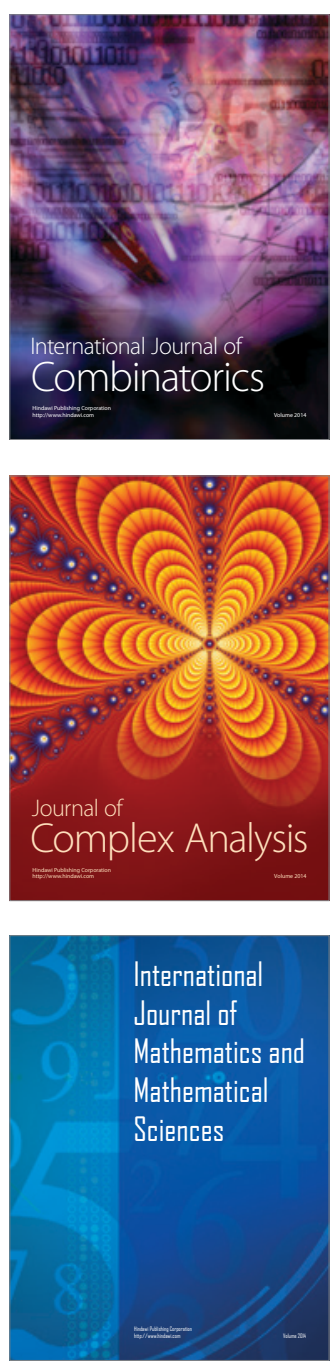
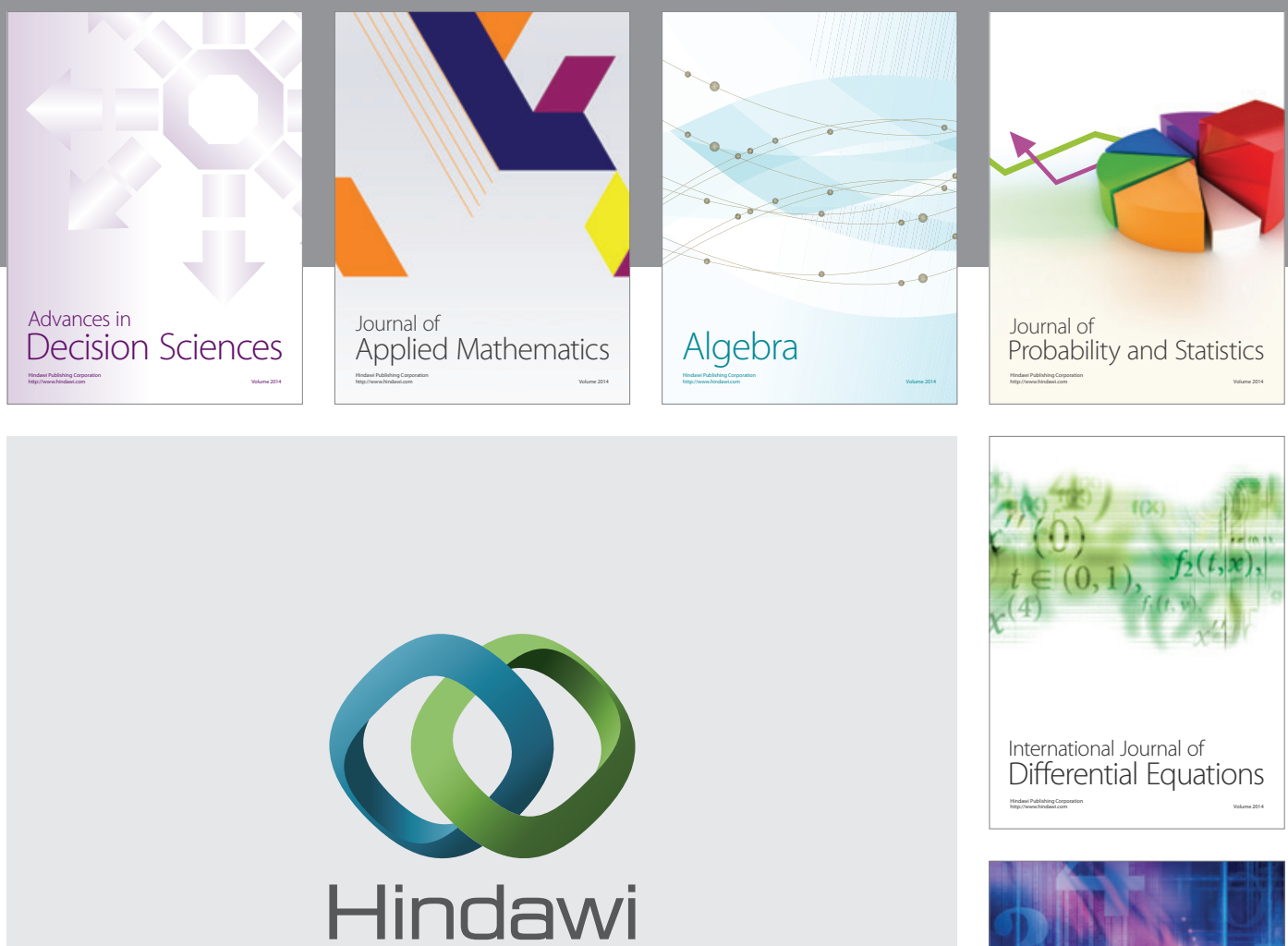

Submit your manuscripts at http://www.hindawi.com
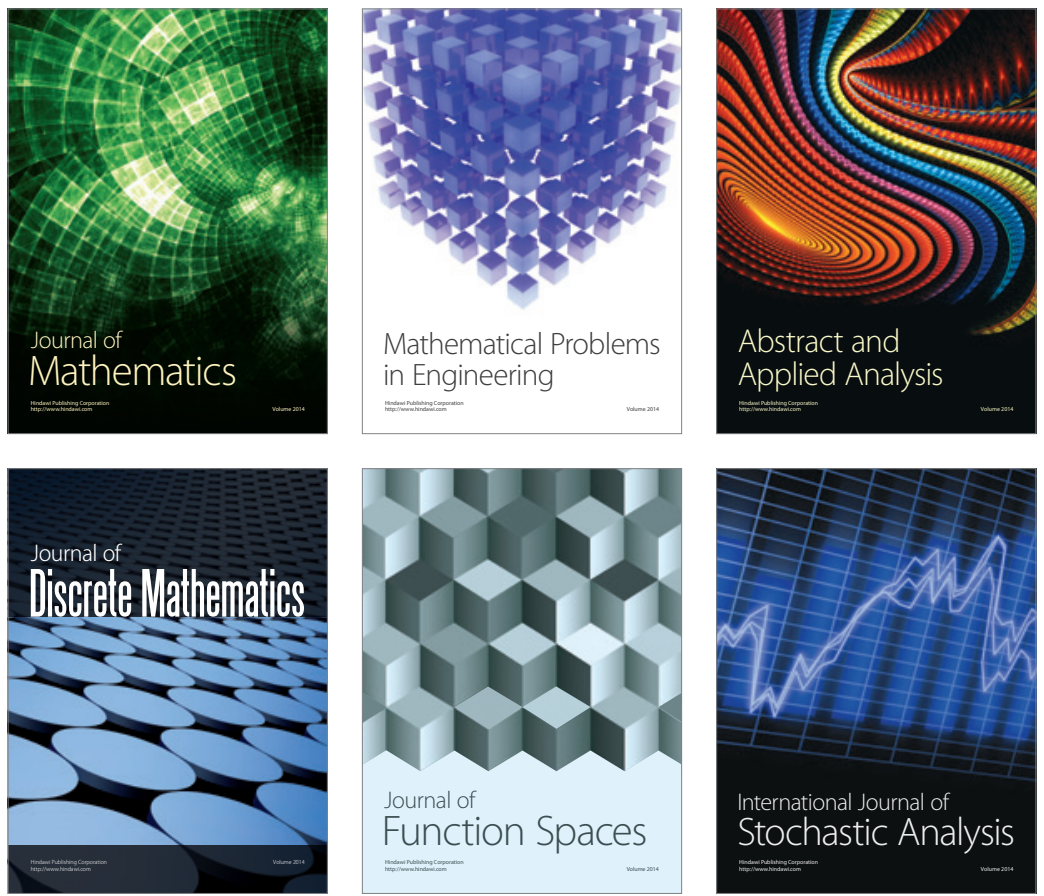

Journal of

Function Spaces

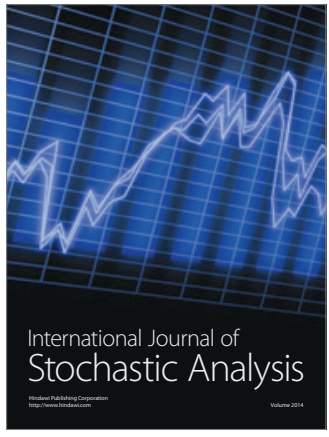

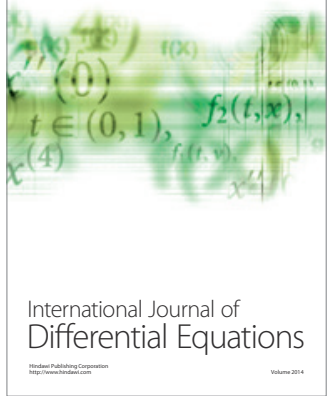
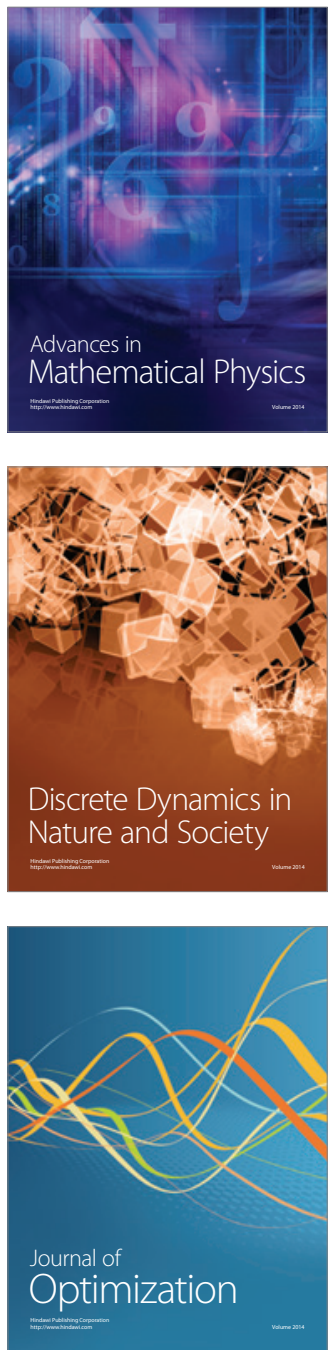